UC3M Working Papers

Statistics and Econometrics

$15-13$

ISSN 2387-0303

June 2015
Departamento de Estadística Universidad Carlos III de Madrid Calle Madrid, 126

28903 Getafe (Spain) Fax (34) 91 624-98-48

\title{
SEASONAL COPULA MODELS FOR THE ANALYSIS OF GLACIER DISCHARGE AT KING GEORGE ISLAND, ANTARCTICA
}

\author{
Gómez, M. ${ }^{\text {, }, ~ A u s i ́ n, ~ M . C . ~}{ }^{\text {, }}$ Domínguez, M. C. ${ }^{\text {b }}$
}

\begin{abstract}
Modelling glacier discharge is an important issue in hydrology and climate research. Glaciers represent a fundamental water resource when melting of snow contributes to runoff. Glaciers are also studied as natural global warming sensors. GLACKMA association has implemented one of their Pilot Experimental Watersheds at the King George Island in the Antarctica which records values of the liquid discharge from Collins glacier. In this paper, we propose the use of time-varying copula models for analyzing the relationship between air temperature and glacier discharge, which is clearly non constant and non linear through time. A seasonal copula model is defined where both the marginal and copula parameters vary periodically along time following a seasonal dynamic. Full Bayesian inference is performed such that the marginal and copula parameters are estimated in a one single step, in contrast with the usual twostep approach. Bayesian prediction and model selection is also carried out for the proposed model such that Bayesian credible intervals can be obtained for the conditional glacier discharge given a value of the temperature at any given time point. The proposed methodology is illustrated using the GLACKMA real data where there is, in addition, a hydrological year of missing discharge data which were not possible to measure accurately due to hard meteorological conditions.
\end{abstract}

Keywords: Bayesian inference; Copulas; Glacier discharge; Seasonality; MCMC; Melt modelling

\footnotetext{
${ }^{\text {a }}$ Department of Statistics, Universidad Carlos III de Madrid.

${ }^{\mathrm{b}}$ Department of Applied Mathematics, Universidad de Salamanca.
} 


\title{
Seasonal copula models for the analysis of glacier discharge at King George Island, Antarctica
}

\author{
Mario Gómez ${ }^{1}$, M. Concepción Ausín ${ }^{1}$ and M. Carmen Domínguez ${ }^{2}$ \\ ${ }^{1}$ Departamento de Estadística, Universidad Carlos III de Madrid, Spain \\ ${ }^{2}$ Departamento de Matemática Aplicada, Universidad de Salamanca, Spain
}

June 10, 2015

\begin{abstract}
Modelling glacier discharge is an important issue in hydrology and climate research. Glaciers represent a fundamental water resource when melting of snow contributes to runoff. Glaciers are also studied as natural global warming sensors. GLACKMA association has implemented one of their Pilot Experimental Watersheds at the King George Island in the Antarctica which records values of the liquid discharge from Collins glacier. In this paper, we propose the use of time-varying copula models for analyzing the relationship between air temperature and glacier discharge, which is clearly non constant and non linear through time. A seasonal copula model is defined where both the marginal and copula parameters vary periodically along time following a seasonal dynamic. Full Bayesian inference is performed such that the marginal and copula parameters are estimated in a one single step, in contrast with the usual two-step approach. Bayesian prediction and model selection is also carried out for the proposed model such that Bayesian credible intervals can be obtained for the conditional glacier discharge given a value of the temperature at any given time point. The proposed methodology is illustrated using the GLACKMA real data where there is, in addition, a hydrological year of missing discharge data which were not possible to measure accurately due to hard meteorological conditions.
\end{abstract}

Keywords: Bayesian inference; Copulas; Glacier discharge; Seasonality; MCMC; Melt modelling;

\section{Introduction}

Glaciers lose mass through different processes such as melting, sublimation and calving. In particular, most of liquid water is lost by surface melting and runoff or surface melting, percolating inside the glacier and exit by the base. Glacier discharge is defined as the rate of flow of meltwater through a vertical section perpendicular 
to the direction of the flow, see e.g. Cogley et al. (2011). Modelling glacier discharge is a very important issue in climate and hydrology research, see e.g. Jansson et al. (2003) and La Frenierre et al. (2014). A review of the different approaches for glacier melt modelling can be found in Hock (2005). These models are usually classified in two main categories: energy balance models, which try to solve balance equations relating the gain and loss of ice in glacier systems, see e.g. Ohmura et al. (1992) and Willis et al. (2002), and temperature index or degree-day models, which are essentially simple linear regression models relating the temperature and the glacier discharge, see e.g. Gray and Prowse (1993), Hock (2003) and Pellicciotti et al. (2005). Temperature-index models are often preferred for their simplicity and because the air temperature is usually easy available. However, the main drawback of these models is that they generally assume that the relation between temperature and glacier discharge is linear and constant along time, which is not realistic in practice.

Copulas have become a common tool to model nonlinear dependencies and nonstationarity. Although the copula concept appears by the late 50s, the computer development in the last decades has caused a fast growth of the number of scientific papers related with copulas, see Nelsen (2006) for an extensive review. The main advantage of copulas is that the marginal distributions can be defined separately from their dependence structure. Copulas have been widely used in many different fields such as a civil engineering, finance, medicine, etc. In particular, in climate and hydrology research, they have been considered for example to model the dependence between the temperature and rainfall, see Scholzel and Friederichs (2008) and Cong and Brady (2012), the relation between intensity and duration of a rainfall, see e.g. Cantet and Arnaud (2014), in characterization of droughts, see e.g. Zhang, et al. (2013), in the analysis of floods, see e.g. Saad et al. (2014), in the behaviour of reconstructed watersheds, see Nazemi and Elshorbagy (2001), spatial models, see e.g. Kazianka and Pilz (2010), among others. See also Genest and Favre (2007) for an introduction to copulas in hydrology. In most of these works, copula models are static such that their parameters remain constant along time. Time-varying copulas have been widely used in finance, see e.g. Patton (2012) and Ausin and Lopes (2010) but their use is very limited in hydrological research.

GLACKMA (Glaciers, Cryokarst and Environment) association promotes scientific research in the polar regions, see http://www.glackma.es. Their researchers have been visiting both poles almost every year since 1985 with the goal of using the glaciers as natural warming sensors, as explained e.g. in Hock et al. (2005) and Bers et al. (2013). GLACKMA has implemented eight stations as Pilot Experimental Watersheds at different latitudes and altitudes in glaciers of both hemispheres which are working continuously to register glacier discharge values. In this paper, we will concentrate on one of these stations located at the King George Island in the Antarctica, where there is available glacier discharge data from 2002. Temperature data are also obtained from the Russian Antarctic Base Bellingshausen, located at $4 \mathrm{~km}$ from the GLACKMA station on King George Island.

The main objective of this paper is to study the time-varying relationship between glacier discharge 

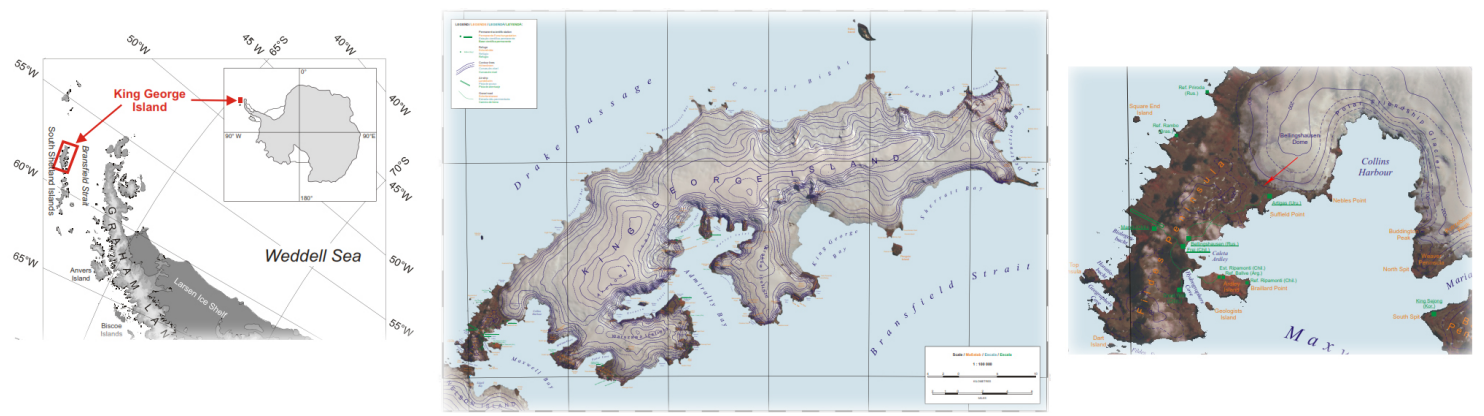

Figure 1: Left panel shows the location of King George Island in the Antarctica. Central panel shows the island, mostly covered by Collins glacier. Right panel zooms in the location of the CPE-KG- $62^{\circ} \mathrm{S}$ station, indicated with a red arrow.

and temperature. We show that the dependence among these two variables is non constant and non linear through time. Therefore, we propose a time-varying seasonal copula model whose parameters follow a seasonal dynamic. The marginal distributions for the discharge and temperature are modelled using timevarying distribution models whose location, scale and shape parameters vary periodically on time. Bayesian inference and prediction is carried out such that it is possible to obtain credible Bayesian intervals for the missing data periods and credible predictive intervals for the glacier discharge value conditioned on the temperature at any given time point whose temperature is known.

This paper is organized as follows. Section 2 describes the study area in Antarctica where the GLACKMA experimental station is installed. A descriptive analysis of the available database is also provided. Section 3 introduces the proposed time-varying copula model where the rank correlations follow a seasonal dynamic and whose marginal distribution parameters also vary periodically along time. Section 4 explains how to undertake Bayesian inference and prediction based on MCMC methods and describes how to perform Bayesian model selection in this context based on DIC. The proposed methodology is applied in Section 5 for the GLACKMA database. Finally, Section 6 concludes with some discussion and extensions.

\section{Study area and data description}

King George Island is the largest of the South Shetland Islands located at the tip of the Antarctic Peninsula. The glaciers of King George Island have suffered a retreat and loss of thickness in the recent decades associated with rising air temperatures, see Rückamp et al. (2011) and Osmanoglu et al. (2013). Collins Glacier, with around $1313 \mathrm{~km}^{2}$, covers most of the King George Island except the south-western end of the island, where the Fildes Peninsula is located. The study area is placed in this south west side of the icecap Collins, known as Smaller Dome or Bellingshausen Dome, see Figure 1.

More specifically, the GLACKMA measuring station, named as CPE-KG- $62^{\circ} \mathrm{S}$, is installed under the 
bridge leading to the Uruguayan Base Artigas $\left(62^{\circ} 1103 \mathrm{~S}, 58^{\circ} 5441 \mathrm{~W}\right)$ where runs a stream bringing water from the unique lagoon which, after diverse proglacial routes, receives the flow from five springs representing the glacier discharge into the south side of the coast. These five springs drain water from a catchment area with a total surface of $2.92 \mathrm{~km}^{2}$, which comprises $1.31 \mathrm{~km}^{2}$ of glacier surface, $0.25 \mathrm{~km}^{2}$ of peripheral moraine and and $1.36 \mathrm{~km}^{2}$ of fluvial surface.

The GLACKMA monitoring station was installed in January of 2002 and consisted of a sounder with sensors for water temperature, conductivity and river level. After two years of hourly registrations, the hard meteorological conditions during the austral winter in 2003 caused a series of invalid records during the following austral summer. A new high-quality sounder was then set up which, although it only registers values from the river level, it is much more resistant under extreme conditions. The glacier discharge can be accurately estimated as an exponential function of the river level using a classical regression fit with $R^{2}=0.99$, see Domínguez and Eraso (2007) for further details.

Therefore, the available discharge data for this project are from October 1st, 2002 to September, 30th 2012, with a missing hydrological year of records from December, 1st 2003 to June, 30th 2004. On the other hand, a complete time series of temperatures is obtained for the whole same period from the weather station of the Russian Antarctic Base Bellingshausen, located at $4 \mathrm{~km}$ from the CPE-KG- $62^{\circ} \mathrm{S}$ station. Daily average values are obtained such that our database consists of a bivariate time series of 3653 observations from two main variables: the average daily glacier discharge, measured in $\mathrm{m}^{3} / \mathrm{s} \cdot \mathrm{km}^{2}$, and the average daily air temperature, measured in Celsius degrees $\left({ }^{\circ} \mathrm{C}\right)$, during ten hydrological years, with 213 consecutive missing values for the discharge.

Figure 2 shows the boxplots of the average daily mean glacier discharge divided by 11-day groups for a smoother description. Note that there is a clear seasonality pattern. First, we observe that during the austral winter, which starts at the end of June, there is almost no glacier discharge. This produces a large amount of zero values which represent around the $57 \%$ of the discharge observations. The period of positive discharge begins at the end of the austral spring, between November and December. During this initial discharge period, there are eventually some extreme values which are usually known as "spring events" or "burst", see Warburton et al (1994), these are brief and violent episodes when the glacier brutally release a large amount of water. They are mostly originated when the bottom of full-of-water vertical wells are broken due to the increase in the temperatures.

Figure 2 also shows that the maximum values for the median discharge are observed during the austral summer, from the end of December to the middle of March. The median discharge starts to decrease with the arrival of the autumn, at the end of March. However, we may also observe extreme values during this period, which are known as "aftershocks", see Warburton et al. (1994). These are kicks in the glacier drainage that may appear when the annual discharge wave seems to be over and are typically caused by the fluctuations 


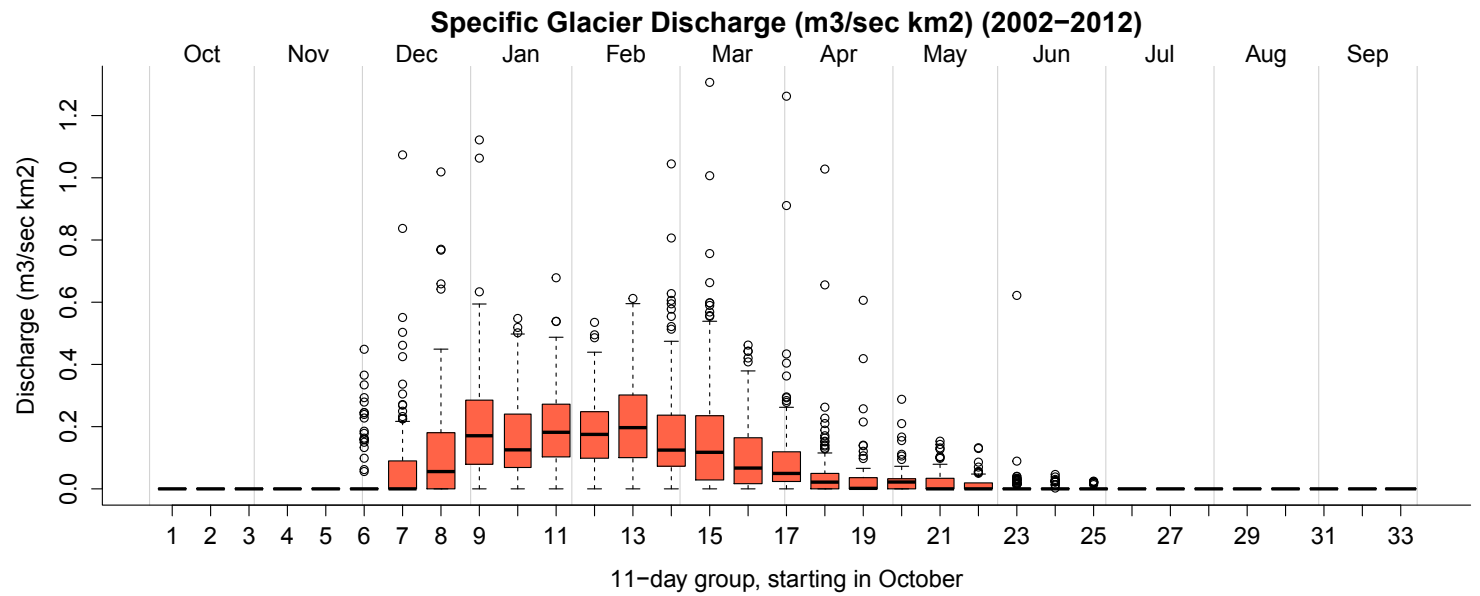

Figure 2: Boxplots of the average daily glacier discharge from 2002 to 2012 divided in 11-day groups.

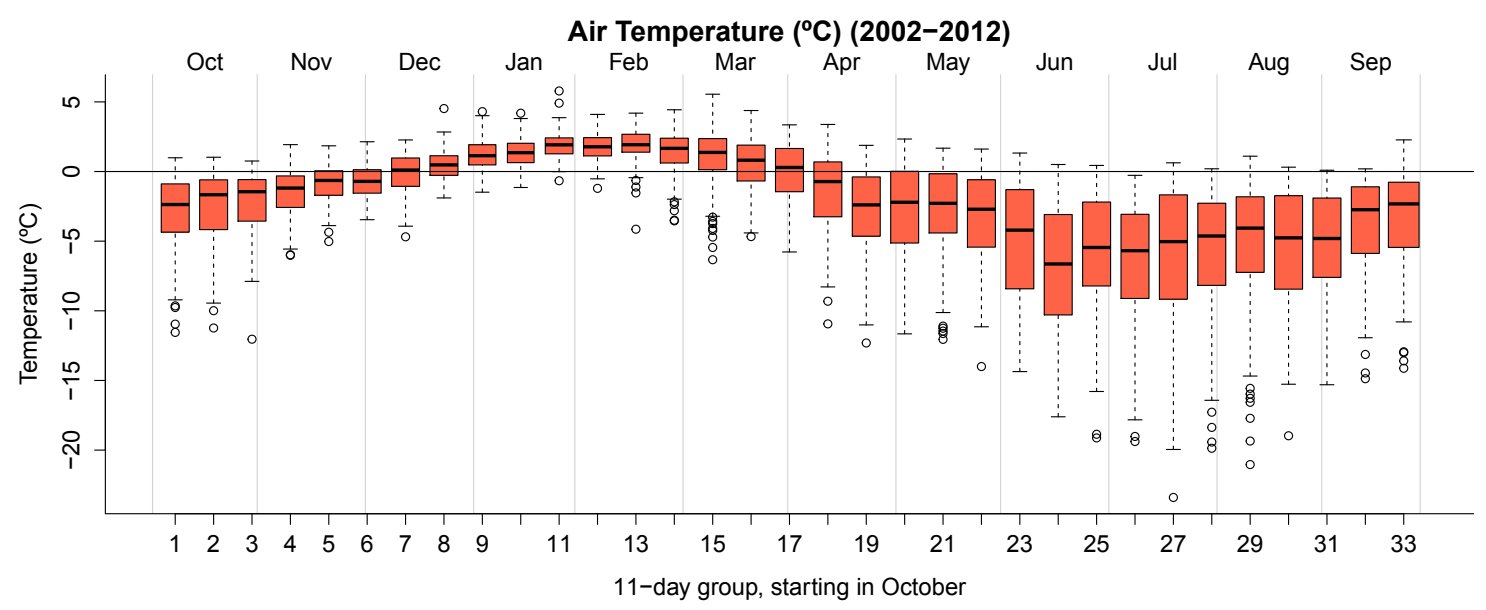

Figure 3: Boxplots of the average daily temperature from 2002 to 2012 divided in 11-day groups.

in the temperature during this period.

Figure 3 shows the boxplots of the average daily temperatures divided again by 11-day groups for a smoother description. As before, we can observe a clear seasonality effect. Note that the average temperatures are above zero only during the austral summer, from the middle of December to the middle of March. Also during the summer period, we can observe less dispersion and more symmetry, in the temperature distribution, than in the rest of the year. On the contrary, temperatures start to decrease with the beginning of autumn and their dispersion increase such that they are almost always below zero during the austral winter, from the end of June to the end of September, when they present a strong left asymmetry. These plots has been obtained with the help of the R package seas, see Toews et al. (2007).

Figures 2 and 3 also show that there is a clear relationship between temperature and glacier discharge. 

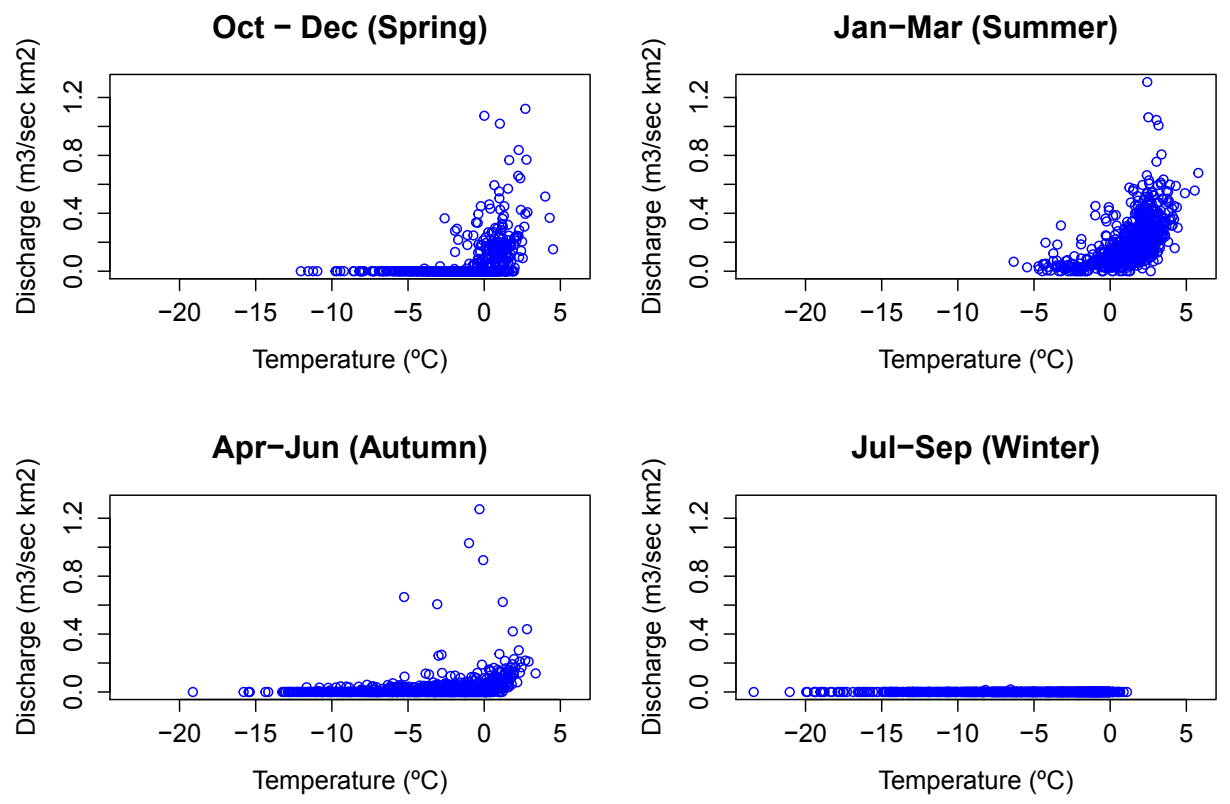

Figure 4: Scatter plots for the daily average temperatures and discharge in each season.

\begin{abstract}
Also, we can observe that this dependence is not constant through time. During the austral winter, when the temperatures are very low, there is no glacier discharge. However, as commented before, the period of positive discharge starts in spring when the temperatures increase and during the austral summer, the median discharge values reach their maximum values due to largest values for temperatures. Therefore, it is clear that there is also a seasonal dynamic in the dependence. This is also illustrated in Figure 4 where the scatterplot for the temperatures and discharges are shown separately for each season. We can observe that there is a strong dependence in summer that disappears in winter. We can also observe that this dependence is not linear.
\end{abstract}

Therefore, in the next section, we propose a model for a time-varying dependence which is not linear and shows a clear seasonal pattern. This is addressed by considering a dynamic joint distribution for the temperature and the glacier discharge whose dependence is measured in terms of a dynamic copula function whose Kendall's tau coefficient moves periodically along time.

\title{
3 Seasonal Model
}

In this section, we present a general model to describe the joint seasonal dynamics for the temperature and the glacier discharge. Firstly, we define separately the marginal models for the temperature and the glacier discharge using time-varying periodic distributions. Then, we describe the seasonal dependence using a time-varying copula model whose parameters vary periodically along time. 


\subsection{Marginals}

Firstly, we define a periodic time series model for the average daily temperature at each day $t$, which will be denoted by $X_{t}$. In order to approximate its seasonal behavior, we assume that the distribution of $X_{t}$ changes periodically through time with a location parameter, $\mu_{t}$, given by:

$$
\mu_{t}=a_{0 \mu}+\sum_{k=1}^{K_{1}} a_{k \mu} \cos \left(\frac{2 k \pi t}{c}-\psi_{k 1}\right),
$$

where $c=365.25$ is the annual periodic cycle. Observe that this is an approximation by a partial sum of a trigonometric Fourier series with $K_{1}$ terms, where the fundamental frequency is $2 \pi / c$, the amplitude parameters are $\boldsymbol{a}_{\mu}=\left(a_{0 \mu}, \ldots, a_{K_{1} \mu}\right)$, where $a_{k \mu} \in \mathbb{R}$, and the phase angle parameters are $\boldsymbol{\psi}_{1}=\left(\psi_{11}, \ldots, \psi_{K_{1} 1}\right)$, where $\psi_{k 1} \in[0, \pi)$. Note that each angle phase, $\psi_{k 1}$, is only defined in the semi-unit circle $[0, \pi)$ since:

$$
a_{k \mu} \cos \left(\frac{2 k \pi t}{c}-\psi_{k 1}\right)=-a_{k \mu} \cos \left(\frac{2 k \pi t}{c}-\left(\psi_{k 1}+\pi\right)\right)
$$

Figure 2 shows that not only the mean of temperature varies periodically along time, but also the variance and possibly, the shape of the distribution. Therefore, we assume similarly that the scale, $\sigma_{t}$, and shape, $\xi_{t}$, parameters of $X_{t}$ vary periodically along time such that,

$$
\begin{aligned}
\log \sigma_{t} & =a_{0 \sigma}+\sum_{k=1}^{K_{1}} a_{k \sigma} \cos \left(\frac{2 k \pi t}{c}-\psi_{k 1}\right) \\
\xi_{t} & =a_{0 \xi}+\sum_{k=1}^{K_{1}} a_{k \xi} \cos \left(\frac{2 k \pi t}{c}-\psi_{k 1}\right)
\end{aligned}
$$

where $\boldsymbol{a}_{\sigma}=\left(a_{0 \sigma}, \ldots, a_{K_{1} \sigma}\right)$ and $\boldsymbol{a}_{\xi}=\left(a_{0 \xi}, \ldots, a_{K_{1} \xi}\right)$ are the amplitude parameters for the scale and shape, respectively, where $a_{k \sigma} \in \mathbb{R}$ and $a_{k \xi} \in \mathbb{R}$. And where $\boldsymbol{\psi}_{1}$ is the same vector of phase parameters defined in (1) for the time-varying location. Note that it makes sense to assume that the phase vector is the same for the location, shape and scale, since we expect the same dynamics for the three parameters such that, for example, when the location increases, the scale and shape decreases. Note also that in (2), we have modelled the logarithm of the scale parameter, $\sigma_{t}$, to avoid that it takes negative values. Therefore, the set of parameters for the temperature is given by $\boldsymbol{\vartheta}_{X}=\left\{\boldsymbol{a}_{\mu}, \boldsymbol{a}_{\sigma}, \boldsymbol{a}_{\xi}, \boldsymbol{\psi}_{1}\right\}$ and the number of Fourier terms, $K_{1}$.

Once we have defined the periodic pattern for the location, scale and shape parameters, it is necessary to specify a distribution model for the time-varying temperature, $X_{t}$. For example, we may assume a skewed normal distribution, $X_{t} \sim S N\left(\mu_{t}, \sigma_{t}, \xi_{t}\right)$, see Azzalini (1985), whose density is given by,

$$
f_{S N}\left(x_{t} \mid \mu_{t}, \sigma_{t}, \xi_{t}\right)=\frac{2}{\sigma_{t}} \phi\left(\frac{x_{t}-\mu_{t}}{\sigma_{t}}\right) \Phi\left(\xi_{t}\left(\frac{x_{t}-\mu_{t}}{\omega_{t}}\right)\right),
$$

where $\phi$ and $\Phi$ denote the pdf and cdf of a standard Gaussian distribution. Note that when $\xi_{t}=0$, we obtain the symmetric normal model, $X_{t} \sim N\left(\mu_{t}, \sigma_{t}\right)$. 
Alternatively, we can consider a generalized extreme value distribution model for the temperature, $X_{t} \sim$ $G E V\left(\mu_{t}, \sigma_{t}, \xi_{t}\right)$, see Embrechts et al. (1997), whose density is given by,

$$
f_{G E V}\left(x_{t} \mid \mu_{t}, \sigma_{t}, \xi_{t}\right)=\frac{1}{\sigma}\left[1+\xi_{t}\left(\frac{x_{t}-\mu_{t}}{\sigma_{t}}\right)\right]^{-\frac{1}{\xi_{t}}-1} \exp \left\{-\left[1+\xi_{t}\left(\frac{x_{t}-\mu_{t}}{\sigma_{t}}\right)\right]^{-\frac{1}{\xi_{t}}}\right\}
$$

for $x_{t}>\mu_{t}-\sigma_{t} / \xi_{t}$ when $\xi_{t}>0$ and for $x_{t}<\mu_{t}-\sigma_{t} / \xi_{t}$ when $\xi_{t}<0$. This is a very flexible distribution which includes the Weibull or the Gumbel distribution as particular cases.

There are many other possibilities that could be considered to model the temperature distribution. In Section 4, we explain how to undertake model selection for the distribution model and for the number of Fourier terms from a Bayesian perspective.

Now, we define a periodic time series model for the average daily discharge at each day $t$, which will be denoted by $Y_{t}$. As before, we approximate the seasonal dynamics for the location, $\lambda_{t}$, and scale, $\beta_{t}$, parameters of $Y_{t}$ using partial sums of Fourier series:

$$
\begin{aligned}
& \log \lambda_{t}=a_{0 \lambda}+\sum_{k=1}^{K_{2}} a_{k \lambda} \cos \left(\frac{2 k \pi t}{c}-\psi_{k 2}\right), \\
& \log \beta_{t}=a_{0 \beta}+\sum_{k=1}^{K_{2}} a_{k \beta} \cos \left(\frac{2 k \pi t}{c}-\psi_{k 2}\right) .
\end{aligned}
$$

where $\boldsymbol{a}_{\lambda}=\left(a_{0 \lambda}, \ldots, a_{K_{2} \lambda}\right), a_{k \lambda} \in \mathbb{R}$, and $\boldsymbol{a}_{\beta}=\left(a_{0 \beta}, \ldots, a_{K_{2} \beta}\right), a_{k \beta} \in \mathbb{R}$, are the amplitude parameters for the location and scale parameters, respectively, and $\boldsymbol{\psi}_{2}=\left(\psi_{12}, \ldots, \psi_{K_{2} 2}\right), \psi_{k 2} \in[0, \pi)$, is the vector of phase parameters. Thus, the vector of parameters for the glacier discharge is given by $\boldsymbol{\vartheta}_{Y}=\left\{\boldsymbol{a}_{\lambda}, \boldsymbol{a}_{\beta}, \boldsymbol{\psi}_{2}\right\}$ and the number of Fourier terms, $K_{2}$.

Clearly, we could also define a similar periodic dynamic for the shape parameter. However, for simplicity, we will only consider positive random variables with two parameters to model the glacier discharge. For example, we may assume a Log-Normal distribution for the glacier discharge whose density is given by,

$$
f_{L N}\left(y_{t} \mid \lambda_{t}, \beta_{t}\right)=\frac{1}{y_{t} \beta_{t} \sqrt{2 \pi}} \exp \left\{-\frac{\left(\log \left(y_{t}\right)-\lambda_{t}\right)^{2}}{2 \beta_{t}^{2}}\right\} \text {. }
$$

Altenatively, we could assume a Gamma distribution, $Y_{t} \sim G\left(\alpha_{t}, \beta_{t}\right)$, whose density is given by:

$$
f_{G}\left(y_{t} \mid \alpha_{t}, \beta_{t}\right)=\frac{\beta_{t}^{\alpha_{t}}}{\Gamma\left(\alpha_{t}\right)} x_{t}^{\alpha_{t}-1} \exp \left\{-\beta_{t} x_{t}\right\}
$$

where the mean, $\lambda_{t}=\alpha_{t} / \beta_{t}$ and scale parameter $\beta_{t}$ are assumed to follow the seasonal dynamics given in (6) and (7), respectively. As commented before, model selection and parameter estimation will be addressed in Section 4.

\subsection{Copula}

As commented in the Introduction, the dependence between the temperature and the glacier discharge is not constant along time. There is a strong dependence between this two variables in the austral summer 
and there is almost no dependence in the austral winter. In order to describe this pattern, in this section we model the dependence between these two variables using a time-varying copula model. More specifically, we assume that the Kendall's tau coefficient, $\tau_{t}$, follows a seasonal dynamic described by a periodic function given by,

$$
\operatorname{logit}\left(\tau_{t}\right)=a_{0 \tau}+\sum_{k=1}^{K_{c}} a_{k \tau} \cos \left(\frac{2 k \pi t}{c}-\psi_{k \tau}\right)
$$

where $\boldsymbol{a}_{\tau}=\left(a_{0 \tau}, \ldots, a_{K_{c}}\right), a_{k \tau} \in \mathbb{R}$, are the amplitude parameters and $\boldsymbol{\psi}_{\tau}=\left(\psi_{1 \tau}, \ldots, \psi_{K_{c} \tau}\right), \psi_{k \tau} \in[0, \pi)$, are the phase parameters of the time-varying tau rank correlation parameter. Thus, the vector of parameters for the copula is given by $\boldsymbol{\vartheta}_{C}=\left\{\boldsymbol{a}_{\tau}, \boldsymbol{\psi}_{\tau}\right\}$ and the number of Fourier terms, $K_{c}$. Observe that by using the logit function, we impose that $\tau_{t}$ is always in the interval $[0,1]$. This makes sense since the dependence between the temperature and the discharge will never be negative.

Different copula models could be used. For example, we might consider that the dependence structure is defined by a time-varying Gumbel copula:

$$
C_{G}\left(u_{t}, v_{t} \mid \theta_{t}\right)=\exp \left\{-\left(\left(-\log u_{t}\right)^{\theta_{t}}+\left(-\log v_{t}\right)^{\theta_{t}}\right)^{-\frac{1}{\theta_{t}}}\right\}
$$

where $u_{t}=F_{X_{t}}\left(x_{t} \mid \vartheta_{X}\right)$ and $v_{t}=F_{Y_{t}}\left(y_{t} \mid \vartheta_{Y}\right)$ are the marginal distribution functions for $X_{t}$ and $Y_{t}$, respectively, at time $t$, and where,

$$
\theta_{t}=\frac{1}{1-\tau_{t}}
$$

where the dynamics of $\tau_{t}$ are specified in (10). One of the main advantages of the Gumbel copula is that it allows for right tail dependence, see Embrechts et al. (2001). Similarly, we could consider many other parametric copula models with time-varying tau correlation, such as the Gaussian copula that do not allow for tail dependence:

$$
C_{G a}\left(u_{t}, v_{t} \mid \theta_{t}\right)=\int_{-\infty}^{\Phi^{-1}\left(u_{t}\right)} \int_{-\infty}^{\Phi^{-1}\left(v_{t}\right)} \frac{1}{2 \pi\left(1-\theta_{t}^{2}\right)^{1 / 2}} \exp \left\{-\frac{x^{2}-2 \theta_{t} x y+y^{2}}{2\left(1-\theta_{t}^{2}\right)}\right\} d x d y,
$$

where $\Phi^{-1}$ denotes the inverse of the distribution function of the univariate standard normal distribution and where,

$$
\theta_{t}=\sin \left(\frac{\pi}{2} \tau_{t}\right)
$$

Another alternative would be to assume a Student-t copula,

$$
C_{S t}\left(u_{t}, v_{t} \mid \theta_{t}, v\right)=\int_{-\infty}^{t_{v}^{-1}\left(u_{t}\right)} \int_{-\infty}^{t_{v}^{-1}\left(v_{t}\right)} \frac{1}{2 \pi\left(1-\theta_{t}^{2}\right)^{1 / 2}}\left\{1+\frac{x^{2}-2 \theta_{t} x y+y^{2}}{v\left(1-\theta_{t}^{2}\right)}\right\}^{-\frac{v+2}{2}} d x d y
$$

where $t_{v}^{-1}$ denotes the inverse of the distribution function of the univariate t distribution with $v$ degrees of freedom and where,

$$
\theta_{t}=\sin \left(\frac{\pi}{2} \tau_{t}\right)
$$

However, this copula model impose symmetric tail dependence, which does not seem realistic in this context, and would also require to estimate the degrees of freedom as an additional parameter. 
Therefore, assuming that the number of terms in each Fourier sum is known, the joint density function for the temperature and the glacier discharge at time $t$ will be given by,

$$
f\left(x_{t}, y_{t} \mid \boldsymbol{\vartheta}_{X}, \boldsymbol{\vartheta}_{Y}, \boldsymbol{\vartheta}_{C}\right)=f_{X_{t}}\left(x_{t} \mid \boldsymbol{\vartheta}_{X}\right) f_{Y_{t}}\left(y_{t} \mid \boldsymbol{\vartheta}_{Y}\right) c\left(F_{X_{t}}\left(x_{t} \mid \boldsymbol{\vartheta}_{X}\right), F_{Y_{t}}\left(y_{t} \mid \boldsymbol{\vartheta}_{Y}\right) \mid \boldsymbol{\vartheta}_{C}\right),
$$

where $f_{X_{t}}$ and $f_{Y_{t}}$ represent the marginal density functions of the glacier discharge and the temperature, respectively, that can be specified for example using the distribution models given in (4) or (5) and (8) or (9), respectively, and where $c$ represents the copula density function whose corresponding cumulative distribution function can be specified for example using (11), (12) or (13).

\section{Inference, prediction and model selection}

Consider now the observed data series, $(\mathbf{x}, \mathbf{y})=\left\{\left(x_{1}, y_{1}\right), \ldots,\left(x_{T}, y_{T}\right)\right\}$, which provides the daily temperature and discharge measurements during $T$ days. Given these data, we would like to make inference on the model parameters, $\boldsymbol{\vartheta}=\left(\boldsymbol{\vartheta}_{X}, \boldsymbol{\vartheta}_{Y}, \boldsymbol{\vartheta}_{C}\right)$. In this section, we first assume that the distribution models for the marginals and the copula are known. Also the number of terms in the Fourier approximations, $K_{1}, K_{2}$ and $K_{c}$, are assumed to be known. Later, in Subsection (4.1), we will explain how to perform Bayesian model selection to select both the distribution models and the number of Fourier terms.

If the data set were complete, the likelihood function would be just the product of the joint density functions, (14), for each $t=1, \ldots, T$. However, as commented in the data description, during the hydrological year 2003/04, it was not possible to register measurements for the glacier discharge since the external datalogger suffered flaws due to the hard meteorological conditions during the winter months. These values will be treated as missing data. Further, there is a large amount of glacier discharge values that are recorded as zero. Considering that the glacier discharge is measured as a function of the level of the river, these zero values can be regarded as left-censored observations since they are actually smaller than a minimum value, $y_{\min }$, below which it is not possible to register any discharge value. The glacier discharge values in these cases are so small that they can not be registered accurately.

Therefore, the likelihood function for the model parameters is given by,

$$
l(\boldsymbol{\vartheta} \mid \mathbf{x}, \mathbf{y})=\prod_{t: y_{t}>0} f\left(x_{t}, y_{t} \mid \boldsymbol{\vartheta}\right) \prod_{t: y_{t}=0} \operatorname{Pr}\left(Y_{t}<y_{\min } \mid x_{t}, \boldsymbol{\vartheta}\right) \cdot f\left(x_{t} \mid \boldsymbol{\vartheta}_{X}\right) \prod_{t: y_{t}=n a} f\left(x_{t} \mid \boldsymbol{\vartheta}_{X}\right),
$$

where $n a$ represents a missing discharge value which is not available and where the conditional probability for the glacier discharge can be obtained as,

$$
\operatorname{Pr}\left(Y_{t}<y_{\min } \mid x_{t}, \boldsymbol{\vartheta}\right)=C^{(1)}\left(F_{X_{t}}\left(x_{t} \mid \boldsymbol{\vartheta}_{X}\right), F_{Y_{t}}\left(y_{\min } \mid \boldsymbol{\vartheta}_{Y}\right) \mid \boldsymbol{\vartheta}_{C}\right),
$$

where $C^{(1)}$ represents the partial derivative of the copula distribution function as described in e.g. Venter $(2001)$,

$$
C^{(1)}\left(u, v \mid \boldsymbol{\vartheta}_{C}\right)=\frac{\partial C_{t}\left(u, v \mid \boldsymbol{\vartheta}_{C}\right)}{\partial u} .
$$


Note that these correspond to the so-called h-functions defined in Aas et al (2009).

For example, for the particular case of a Gumbel copula, it is obtained that,

$$
C_{G}^{(1)}\left(u_{t}, v_{t} \mid \theta_{t}\right)=C_{G}\left(u_{t}, v_{t} \mid \theta_{t}\right)\left[\left(-\ln u_{t}\right)^{\theta_{t}}+\left(-\ln v_{t}\right)^{\theta_{t}}\right]^{-1+\frac{1}{\theta_{t}}}\left(-\ln u_{t}\right)^{\theta_{t}-1} \frac{1}{u_{t}},
$$

where $C_{G}\left(u_{t}, v_{t} \mid \theta_{t}\right)$ is the Gumbel copula distribution function given in (11). And for the Gaussian copula, the $C^{(1)}$ function can be expressed as

$$
C_{G a}^{(1)}\left(u_{t}, v_{t} \mid \theta_{t}\right)=\Phi\left(\Phi^{-1}\left(v_{t}\right) \mid \theta_{t} \cdot \Phi^{-1}\left(u_{t}\right), 1-\theta_{t}^{2}\right),
$$

where $\Phi\left(x \mid \mu, \sigma^{2}\right)$ denotes the Gaussian density function with mean $\mu$ and variance $\sigma^{2}$, and $\Phi(x)$ denotes the standard Gaussian density function.

In order to perform Bayesian inference, we must define prior distributions for the model parameters, $\boldsymbol{\vartheta}$. We impose proper but non informative prior distributions as follows. For each amplitude parameter, $a_{k p}$, we assume a large variance Gaussian prior $N\left(0,100^{2}\right)$, for $k=0, \ldots, K_{j}$, for $j=1,2, c$ and for $p=\mu, \sigma, \xi, \lambda, \beta, \tau$. For each phase parameter, $\psi_{k j}$, we assume a uniform semicircular variable in $[0, \pi)$, for $k=0, \ldots, K_{j}$ and for $j=1,2, c$.

Given these priors and the likelihood specified in (15), it is not straightforward to derive analytically the posterior distribution, $f(\boldsymbol{\vartheta} \mid \mathbf{x}, \mathbf{y})$. Therefore, we use MCMC sampling strategies in order to obtain a sample from the joint posterior distribution of the parameters, which allows us to develop Bayesian inference. We propose a Gibbs sampling scheme which is carried out by cycling repeatedly through draws of each parameter conditional on the remaining parameters, see Tierney (1994). In particular, we use the Random Walk Metropolis Hastings (RWMH) algorithm for sampling from the conditional posterior distribution of the model parameters. We consider a simple one-dimensional RWMH where each model parameter is updated separately using normal candidate distributions whose mean is given by the previous value of each parameter in the algorithm and whose variance can be calibrated to obtain good acceptance rates. The details of the proposed algorithm are explained in the Appendix.

Now, we are interested in estimating the predictive joint distribution of the temperature and discharge, $f\left(x_{t}, y_{t} \mid \mathbf{x}, \mathbf{y}\right)$, at any time $t$. This can be done using Monte Carlo simulation based on the MCMC output. Consider a posterior sample of size $M$ of the model parameters, $\boldsymbol{\vartheta}^{(i)}$, for $i=1, \ldots, M$. Then, the values of the time-varying parameters, $\mu_{t}^{(i)}, \sigma_{t}^{(i)}, \xi_{t}^{(i)}, \lambda_{t}^{(i)}, \beta_{t}^{(i)}$ and $\tau_{t}^{(i)}$, are known for each time $t$ and we can simulate values from $f\left(x_{t}, y_{t} \mid \mathbf{x}, \mathbf{y}\right)$ as follows.

For each $t=1, \ldots, T$ and $i=1, \ldots, M$.

1. Obtain the copula parameter $\theta_{t}^{(i)}$ from $\tau_{t}^{(i)}$

2. Simulate a value from the copula: $\left(u_{t}^{(i)}, v_{t}^{(i)}\right) \mid \theta_{t}^{(i)}$ 
3. Obtain the pair of values for the temperature and discharge:

$$
x_{t}^{(i)}=F_{X}^{-1}\left(u_{t}^{(i)} \mid \mu_{t}^{(i)}, \sigma_{t}^{(i)}, \xi_{t}^{(i)}\right), \quad y_{t}^{(i)}=F_{Y}^{-1}\left(v_{t}^{(i)} \mid \lambda_{t}^{(i)}, \beta_{t}^{(i)}\right) .
$$

Given this sample of the joint posterior distribution, we can obtain a sample from the marginal predictive distribution of the temperature by just taking the values $\left\{\left(x_{1}^{(i)}, \ldots, x_{T}^{(i)}\right)\right\}_{i=1}^{M}$. The posterior predictive mean and $95 \%$ credible predictive intervals can be approximated using the sample mean for each $t$ and the corresponding 0.025 and 0.975 quantiles. Similarly, we can approximate the posterior predictive mean and predictive intervals for the glacier discharge.

Finally, we wish to estimate the conditional predictive distribution of the glacier discharge given a value for the temperature, $f\left(y_{t} \mid X_{t}=x_{t}, \mathbf{x}, \mathbf{y}\right)$, at any time $t$. As before, this can be done by Monte Carlo approximation given the MCMC output as follows.

For each $t=1, \ldots, T$ and $i=1, \ldots, M$,

1. Obtain $u_{t}^{(i)}=F_{X}\left(x_{t} \mid \mu_{t}^{(i)}, \sigma_{t}^{(i)}, \xi_{t}^{(i)}\right)$ from the distribution selected for the temperature,

2. Find $v_{t}^{(i)}$ such that $p=C^{(1)}\left(u_{t}^{(i)}, v_{t}^{(i)} \mid \theta_{t}^{(i)}\right)$ where $p \sim U(0,1)$

3. Set $y_{t}^{(i)}=F_{Y}^{-1}\left(v_{t}^{(i)} \mid \lambda_{t}^{(i)}, \beta_{t}^{(i)}\right)$

Therefore, given a set of observed temperatures, $\left\{x_{1}, \ldots, x_{T}\right\}$, we can obtain a sample of the conditional predictive distribution of the discharge for each time point, $\left\{\left(y_{1}^{(i)}, \ldots, y_{T}^{(i)}\right)\right\}_{i=1}^{M}$. Using this sample, we can estimate the posterior predictive mean and $95 \%$ credible predictive intervals for the conditional discharge using the sample means and the 0.025 and 0.975 quantiles of the sample as before.

\subsection{Model selection}

In order to compare different models, we use de Deviance Information Criterion (DIC). Models with smaller DIC should be preferred to models with larger DIC, see Spiegelhalter et al. (2002). This measure penalizes the effective number of parameters of the model. The DIC value is given by,

$$
D I C=-4 E_{\vartheta}[\log l(\boldsymbol{\vartheta} \mid \mathbf{x}, \mathbf{y}) \mid x, y]+2 \log l\left(E_{\vartheta}[\boldsymbol{\vartheta} \mid \mathbf{x}, \mathbf{y}] \mid \mathbf{x}, \mathbf{y}\right)
$$


where the $\log$-likelihood of the model parameters, $\boldsymbol{\vartheta}=\left(\boldsymbol{\vartheta}_{X}, \boldsymbol{\vartheta}_{Y}, \boldsymbol{\vartheta}_{C}\right)$, is given by:

$$
\begin{aligned}
\log l(\boldsymbol{\vartheta} \mid \mathbf{x}, \mathbf{y}) & =\sum_{t} \log \left(f_{X}\left(x_{t} \mid \boldsymbol{\vartheta}_{X}\right)\right) \\
& +\sum_{t: y_{t}>0} \log \left(f_{Y}\left(y_{t} \mid \boldsymbol{\vartheta}_{Y}\right)\right) \\
& +\sum_{t: y_{t}>0} \log \left(c\left(F_{X}\left(x_{t} \mid \boldsymbol{\vartheta}_{X}\right), F_{Y}\left(y_{t} \mid \boldsymbol{\vartheta}_{Y}\right) \mid \boldsymbol{\vartheta}_{C}\right)\right) \\
& +\sum_{t: y_{t}=0} \log \left(C^{1}\left(F_{X}\left(x_{t} \mid \boldsymbol{\vartheta}_{X}\right), F_{Y}\left(y_{\text {min }} \mid \boldsymbol{\vartheta}_{Y}\right) \mid \boldsymbol{\vartheta}_{C}\right)\right)
\end{aligned}
$$

Given an MCMC sample of size $M$ of the posterior distribution of the model parameters, $\boldsymbol{\vartheta}^{(i)}$, for $i=1, \ldots, M$, the DIC value, (16), can be approximated by,

$$
D I C=-\frac{4}{M} \sum_{i=1}^{M} \log l\left(\boldsymbol{\vartheta}^{(m)} \mid x, y\right)+2 \log l\left(\frac{1}{M} \sum_{i=1}^{M} \vartheta^{(m)} \mid x, y\right),
$$

\section{Results}

In this section, we illustrate the proposed methodology with the real data provided by GLACKMA on the discharge and temperature measurements from October 1st, 2002 to September 30th, 2012. We have considered a large number of different models for the marginal and copula distributions that will be discussed later in Subsection 5.1. Here, we present firstly the results for the preferred model according to the DIC criteria which consists of a GEV model, see (5), for the marginal distribution of the temperature with $K_{1}=4$ Fourier components, a Gamma distribution, see (9), for the marginal distribution of the discharge with $K_{2}=4$ Fourier components and Gumbel copula, see (11), with $K_{c}=2$ Fourier componets.

The proposed MCMC algorithm is run for 100000 iterations, discarding the first 50000 as burn-in iterations. The chains have converged and they have good mixing. Table 1 shows the mean, posterior deviation and $95 \%$ credible intervals for the model parameters.

Figure 5 shows the real discharge time series data, the posterior predictive means and $95 \%$ credible intervals for the whole time period. Apparently the discharge is well modeled, for example the posterior means are very close to zero in those winter periods where no discharge is recorded and the length of the corresponding credible intervals are also very close to zero. On the contrary, during the summer periods, the posterior discharge means and credible intervals are far from zero. Also, we can observe that the proposed model captures the Spring events and aftershocks at the beginning and the end of each period, respectively.

Finally, observe that the proposed method is also able to produce Bayesian estimates and credible intervals for the missing period during the hydrological year 2003/2004.

Figure 6 shows the real temperature time series, the posterior predictive means and $95 \%$ credible intervals 


\begin{tabular}{|c|c|c|c|c|c|c|c|c|}
\hline \multicolumn{3}{|c|}{ Temperature } & \multicolumn{3}{|c|}{ Discharge } & \multicolumn{3}{|c|}{ Copula } \\
\hline & mean (sd) & int.cred. & & mean (sd) & int.cred. & & mean (sd) & int.cred. \\
\hline$\psi_{11}$ & $2.016(0.017)$ & $(1.983,2.050)$ & $\psi_{12}$ & $2.731(0.027)$ & $(2.674,2.783)$ & $\psi_{1 \tau}$ & $2.269(0.482)$ & $(0.785,2.830)$ \\
\hline$\psi_{21}$ & 2.943 (0.098) & $(2.742,3.117)$ & $\psi_{22}$ & $2.841(0.040)$ & $(2.765,2.920)$ & $\psi_{2 \tau}$ & $1.588(0.814)$ & $(0.109,3.019)$ \\
\hline$\psi_{31}$ & $2.865(0.134)$ & $(2.594,3.107)$ & $\psi_{32}$ & $2.783(0.074)$ & $(2.639,2.926)$ & $a_{1 \tau}$ & $0.821(0.395)$ & $(0.163,1.673)$ \\
\hline$\psi_{41}$ & $0.646(0.154)$ & $(0.363,0.982)$ & $\psi_{42}$ & 2.916 & $(2.573,3.132)$ & $a_{2 \tau}$ & $-0.195(0.216)$ & $(-0.578,0.280)$ \\
\hline$a_{1 \mu}$ & $4.002(0.075)$ & $(3.855,4.152)$ & $a_{1 \lambda}$ & $6.692(0.668)$ & $(5.604,7.940)$ & $a_{0 \tau}$ & $-1.163(0.291)$ & $(-1.769,-0.628)$ \\
\hline$a_{2 \mu}$ & $-0.265(0.071)$ & $(-0.407,-0.126)$ & $a_{2 \lambda}$ & 3.378 (0.433) & $(2.654,4.274)$ & & & \\
\hline$a_{3 \mu}$ & $-0.325(0.063)$ & $(-0.456,-0.207)$ & $a_{3 \lambda}$ & $1.638_{(0.232)}$ & $(1.231,2.174)$ & & & \\
\hline$a_{4 \mu}$ & $-0.152(0.062)$ & $(-0.274,-0.029)$ & $a_{4 \lambda}$ & $0.447(0.091)$ & $(0.279,0.644)$ & & & \\
\hline$a_{0 \mu}$ & $-2.777(0.056)$ & $(-2.886,-2.665)$ & $a_{0 \lambda}$ & $-6.431(0.406)$ & $(-7.179,-5.749)$ & & & \\
\hline$a_{1 \sigma}$ & $-0.721(0.018)$ & $(-0.757,-0.687)$ & $a_{1 \beta}$ & $-0.889(0.415)$ & $(-1.653,-0.108)$ & & & \\
\hline$a_{2 \sigma}$ & $-0.136(0.019)$ & $(-0.173,-0.100)$ & $a_{2 \beta}$ & $-1.528(0.225)$ & $(-1.971,-1.093)$ & & & \\
\hline$a_{3 \sigma}$ & $0.102(0.020)$ & $(0.063,0.142)$ & $a_{3 \beta}$ & $-1.783(0.168)$ & $(-2.120,-1.459)$ & & & \\
\hline$a_{4 \sigma}$ & $0.014(0.016)$ & $(-0.018,0.045)$ & $a_{4 \beta}$ & $-0.432(0.116)$ & $(-0.665,-0.210)$ & & & \\
\hline$a_{0 \sigma}$ & 0.975 (0.013) & $(0.949,1.002)$ & $a_{0 \beta}$ & $2.302(0.273)$ & $(1.786,2.795)$ & & & \\
\hline$a_{1 \xi}$ & $0.261(0.014)$ & $(0.233,0.289)$ & & & & & & \\
\hline$a_{2 \xi}$ & $0.114(0.016)$ & $(0.081,0.145)$ & & & & & & \\
\hline$a_{3 \xi}$ & $0.004(0.014)$ & $(-0.025,0.032)$ & & & & & & \\
\hline$a_{4 \xi}$ & -0.029 & $(-0.05,-0.008)$ & & & & & & \\
\hline$a_{0 \xi}$ & $-0.471(0.009)$ & $(-0.488,-0.454)$ & & & & & & \\
\hline
\end{tabular}

Table 1: Model parameters for the parameters of the GEV distribution for the temperature, the Gamma distribution for the discharge and the rank-tau for the copula. Each parameter is obtained as the mean of its MCMC. The posterior deviation is the number between parenthesis. The third column of each parameter are the credible intervals. 


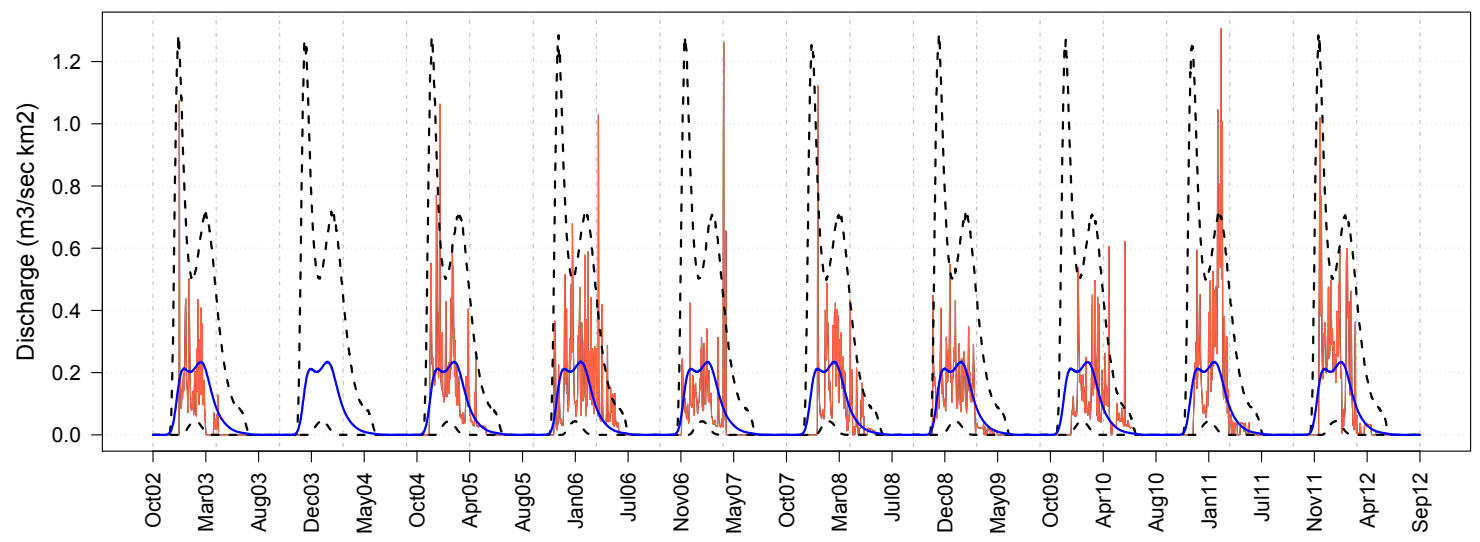

Figure 5: Real discharge data, posterior predictive means and $95 \%$ credible intervals.

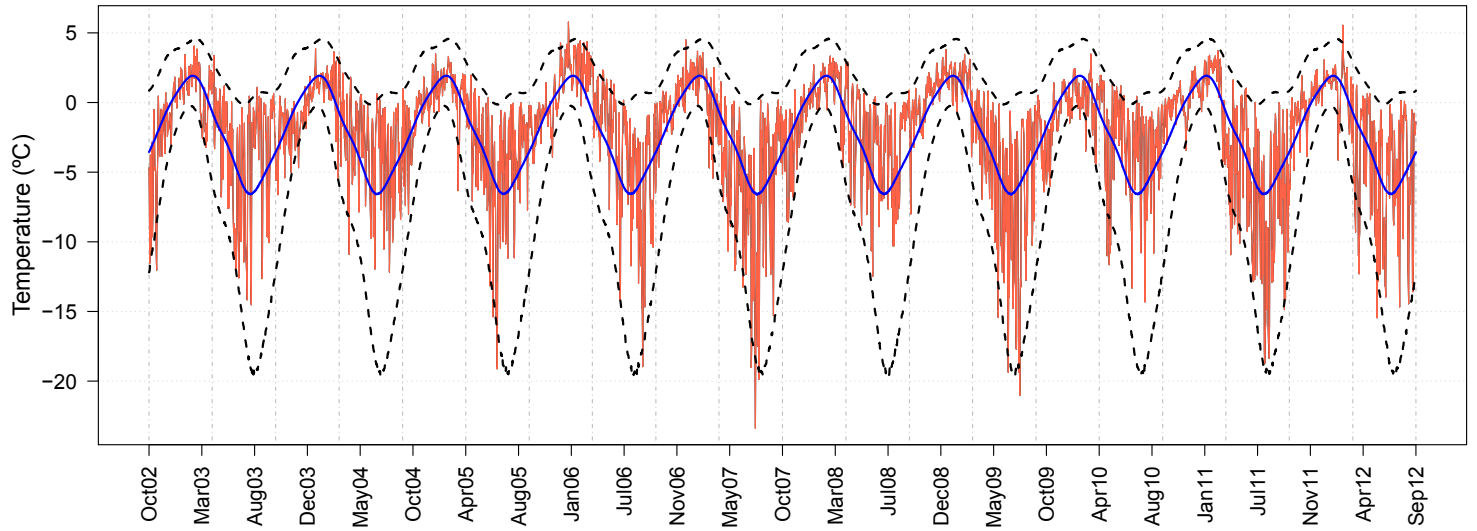

Figure 6: Real temperature data, posterior predictive means and 95\% credible intervals.

for the whole time period. Observe that the model can capture the left-skewness and larger variability during the austral winter. In contrast, note that credible intervals are more symmetric and narrower during the summer periods.

Now, we are interested in analyzing the influence of the temperature on the discharge. Observe that using our proposed approach, we can obtain estimations of the conditional predictive distribution of the discharge given any value of the temperature at any given time point. As an illustration, Figure 7 shows the conditional density function of the discharge for one particular day of the austral summer (02/20/2006) given different values of the temperature. Note that, as expected, the larger is the temperature, the larger is the probability of observing large values for the glacier discharge. Using the same approach, Figure 8 shows the Bayesian estimations of the missing discharge values conditioned on the observed values for the temperature during the hydrological year 2003/2004 when the data-logger did not record the data appropriately. 


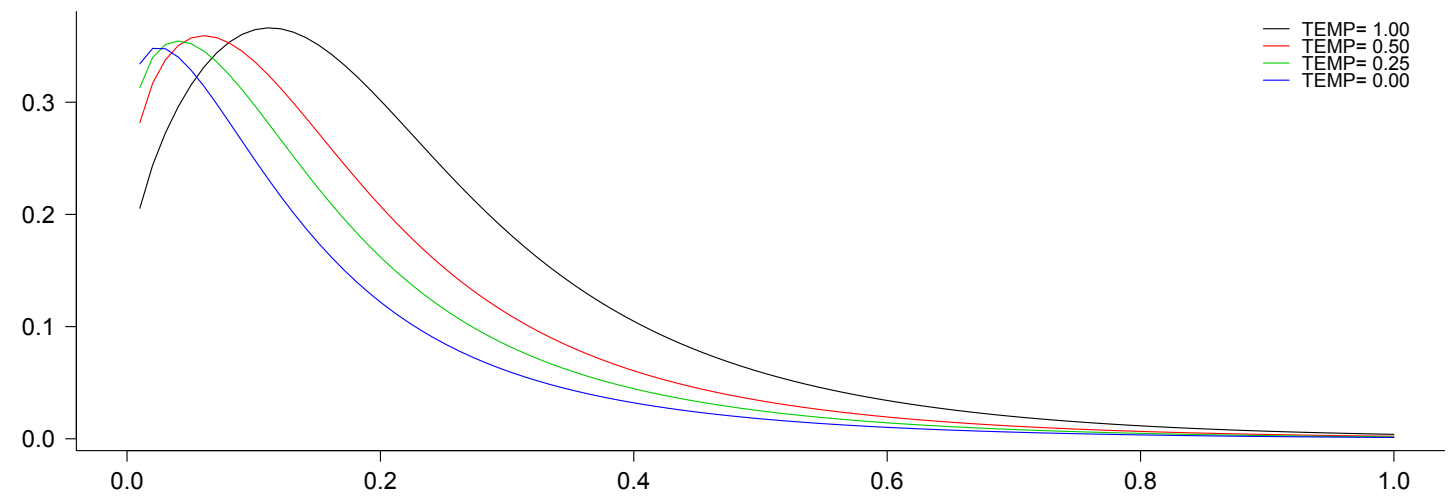

Figure 7: Conditional predictive density of the discharge given different values of the temperature for one particular day in summer $(02 / 20 / 2006)$.

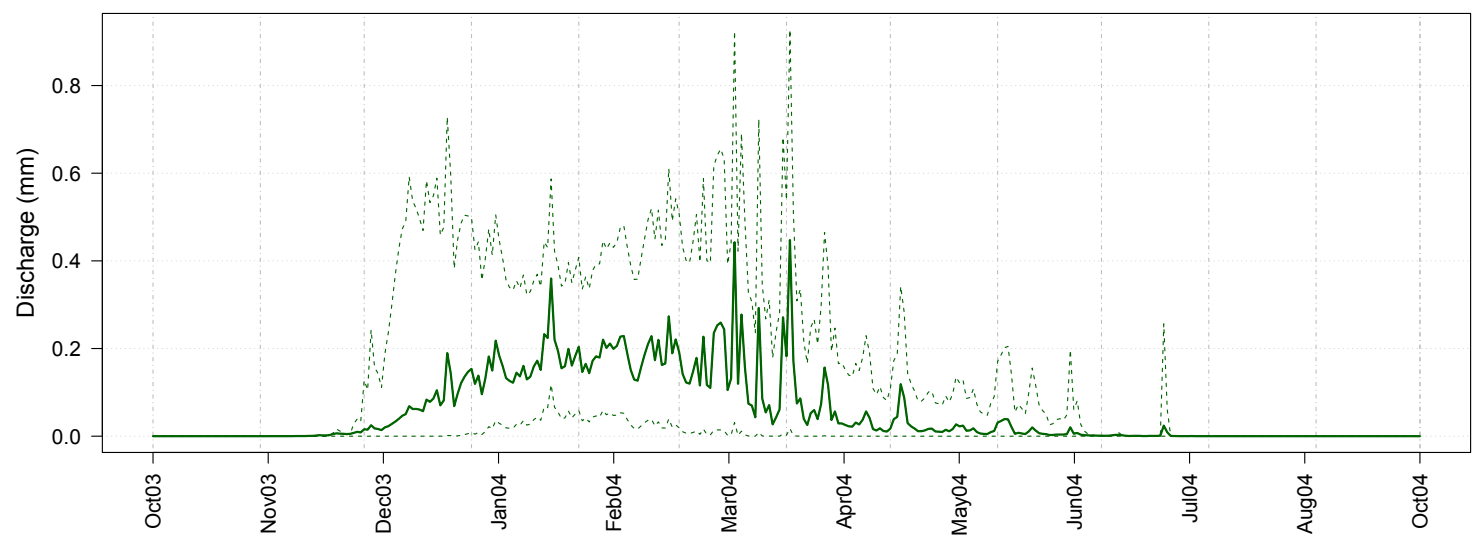

Figure 8: Predicted values for the missing discharge conditioned on the observed values for the temperature during the hydrological year 2003/2004. Dotted lines represent the $95 \%$ credible intervals. 


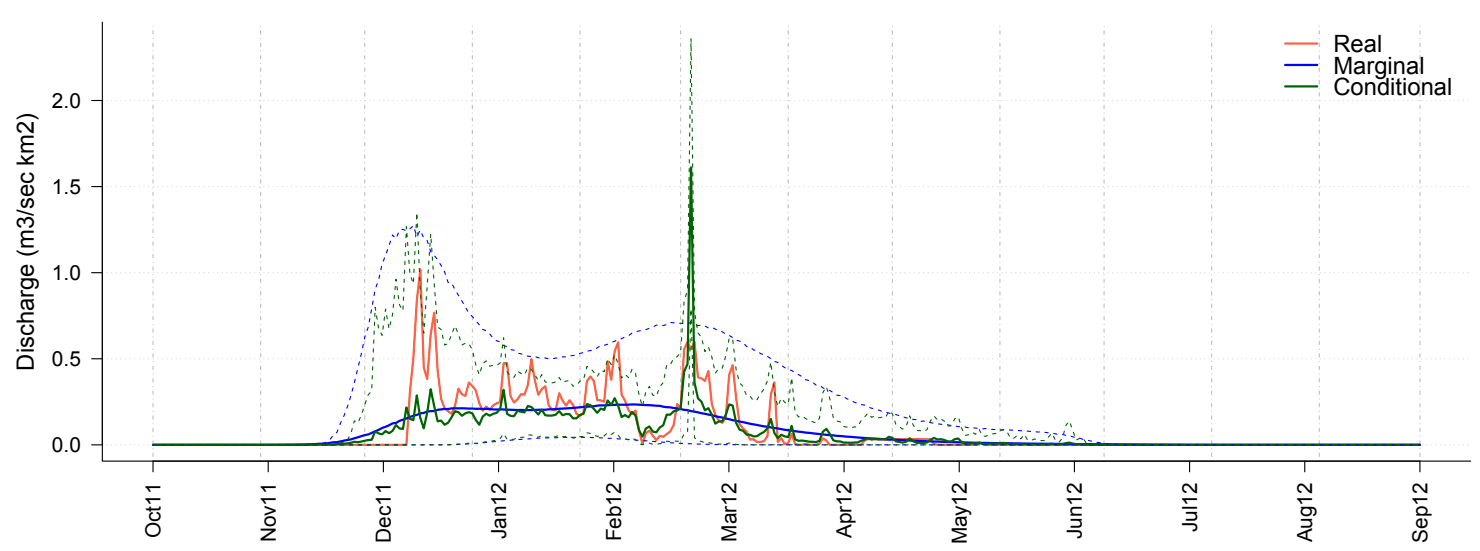

Figure 9: Real data for the discharge, mean of the values of the predictive and $95 \%$ credible intervals. Hydrological year 2011-12

Finally, observe that our proposed methodology also allows for future predictions of both the joint distribution of discharge and temperature and for the conditional discharge distribution given the temperature values. In order to illustrate this, Figure 9 shows the estimations of the predictive discharge distribution for the last hydrological year 2011/2012 given the information from previous years. These are compared with the true observed values during this year. Note that the predictive intervals always contain the true observed values. Figure 9 also shows the estimations of the conditional predictive discharge during this last year given the values for the temperature. Observe that this provides in general better estimations for the discharge, although there is one single day where the temperature was extremely high which leads a large estimation for discharge.

\subsection{Model selection}

In this section, we illustrate how the model introduced before has been selected according to the DIC criteria. Firstly, we put the emphasis on selecting the number of Fourier terms for the time-varying parameters of the temperature, $K_{1}$, the discharge, $K_{2}$ and the copula, $K_{c}$. Table 2 shows the DIC values for different choices of the number of Fourier terms assuming a Generalized Extreme Value for the temperature, a Gamma distribution for the discharge and a Gumbel copula for the dependence. Note that the minimum value corresponds to $K_{1}=4$ terms for the temperature, $K_{2}=4$ terms for the discharge and $K_{c}=2$ terms for the time-varying $\tau_{t}$ parameter of the copula.

Similar tables have been obtained assuming different models for the marginal distributions of the temperature and the discharge and also for the copula. The number of selected Fourier terms is in general the same but the value of the DIC is larger in all cases. For example, the minimum DIC value assuming a 


\begin{tabular}{cccc|cccc}
\hline$K_{1}$ & $K_{2}$ & $K_{c}$ & $D I C$ & $K_{1}$ & $K_{2}$ & $K_{c}$ & $D I C$ \\
\hline 4 & 4 & 2 & 14825 & 1 & 4 & 1 & 15043 \\
4 & 4 & 1 & 14833 & 1 & 4 & 2 & 15044 \\
4 & 4 & 4 & 14843 & 1 & 3 & 3 & 15115 \\
4 & 4 & 3 & 14861 & 1 & 3 & 1 & 15135 \\
3 & 4 & 1 & 14872 & 1 & 3 & 2 & 15178 \\
2 & 4 & 1 & 14921 & 1 & 2 & 1 & 15259 \\
1 & 4 & 3 & 15036 & 1 & 1 & 1 & 15750 \\
\hline
\end{tabular}

Table 2: DIC values for different number of Fourier terms, $K_{1}, K_{2}$ and $K_{c}$, assuming a GEV distribution for the temperature, a Gamma distribution for the discharge and Gumbel copula.

Gaussian copula and the same marginal distribution models as before is $D I C=21723$. Note that this value is larger than the minimum value obtained in Table 2 with a Gumbel Gumbel Copula which is given by $D I C=14825$, indicating that the Gumbel model is preferred than the Gaussian copula.

\section{Conclusion and extensions}

In this paper, we have proposed a seasonal dynamic model to describe the joint distribution of the glacier discharge and air temperature where not only the marginal distributions are time varying but also, the relationship between these two variables is described by a time-varying copula. We have proposed a Bayesian procedure for inference on the model parameters and prediction of the joint discharge and temperature distribution. Our approach allows for the simultaneous estimation of the marginal and copula parameters, which is in contrast with the classical two-stage estimation procedures.

The proposed procedure could be extended to a multivariate model by including more environmental variables like precipitation, humidity, solar radiation or atmospheric pressure. In this case, the use of multivariate copulas would be required. One possibility is the use of vine copulas that has been successfully applied for financial time series data, see Aas et al. (2009). See also Czado (2010) for a survey of vine copulas.

The developed methodology could be also applied in other Pilot Experimental Watersheds installed by GLACKMA at different latitudes in both hemispheres, which could be compared with those obtained in this work. 


\section{Appendix: Algorithm}

In this appendix, we explain in detail the proposed MCMC algorithm to sample from the posterior distribution of the model parameters, $\boldsymbol{\vartheta}=\left(\boldsymbol{\vartheta}_{X}, \boldsymbol{\vartheta}_{Y}, \boldsymbol{\vartheta}_{C}\right)$. Recall that the loglikelihood is given by:

$$
\begin{aligned}
\log l(\boldsymbol{\vartheta} \mid \mathbf{x}, \mathbf{y}) & =\sum_{t} \log \left(f_{X}\left(x_{t} \mid \boldsymbol{\vartheta}_{X}\right)\right) \\
& +\sum_{t: y_{t}>0} \log \left(f_{Y}\left(y_{t} \mid \boldsymbol{\vartheta}_{Y}\right)\right) \\
& +\sum_{t: y_{t}>0} \log \left(c\left(F_{X}\left(x_{t} \mid \boldsymbol{\vartheta}_{X}\right), F_{Y}\left(y_{t} \mid \boldsymbol{\vartheta}_{Y}\right) \mid \boldsymbol{\vartheta}_{C}\right)\right) \\
& +\sum_{t: y_{t}=0} \log \left(C_{t}^{1}\left(F_{X}\left(x_{t} \mid \boldsymbol{\vartheta}_{X}\right), F_{Y}\left(y_{\min } \mid \boldsymbol{\vartheta}_{Y}\right) \mid \boldsymbol{\vartheta}_{C}\right)\right)
\end{aligned}
$$

We construct a Gibbs sampling scheme where each model parameter is updated separately. Therefore, it is not necessary to compute the whole likelihood for each parameter. In particular, when updating the parameters corresponding to the temperature, $\boldsymbol{\vartheta}_{X}$, it is only necessary to consider (22), (24) and (25). When updating the discharge parameters, $\boldsymbol{\vartheta}_{Y}$, only (23), (24) and (25) are evaluated. And finally, for updating the copula parameters, $\boldsymbol{\vartheta}_{C}$, only (24) and (25) are considered.

The structure of the proposed MCMC method is shown in Algorithm 1. Firstly, it is required to set a vector of initial values for the parameters and the number of MCMC iterations. Then, in each step of the algorithm, each model parameter is updated using a RWMH which is defined in Algorithm 2. Observe that the algorithm is written such that it is not necessary to recalculate the likelihood that was evaluated in previous step for accepted parameters. Finally, Algorithms 3, 4 and 5 separates the computation of the likelihood as the sum of the log-likelihood temperature, discharge and copula, respectively. 


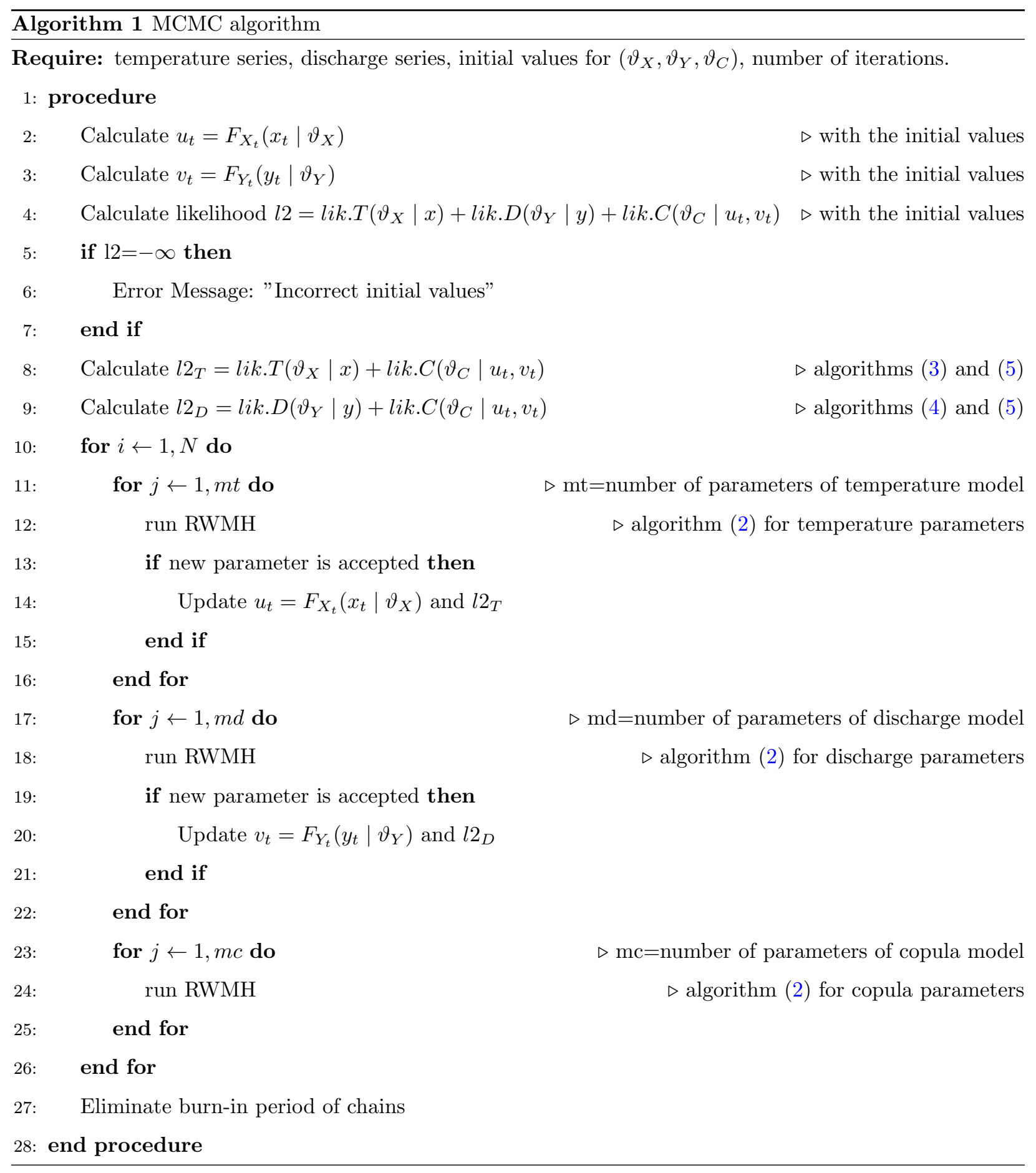




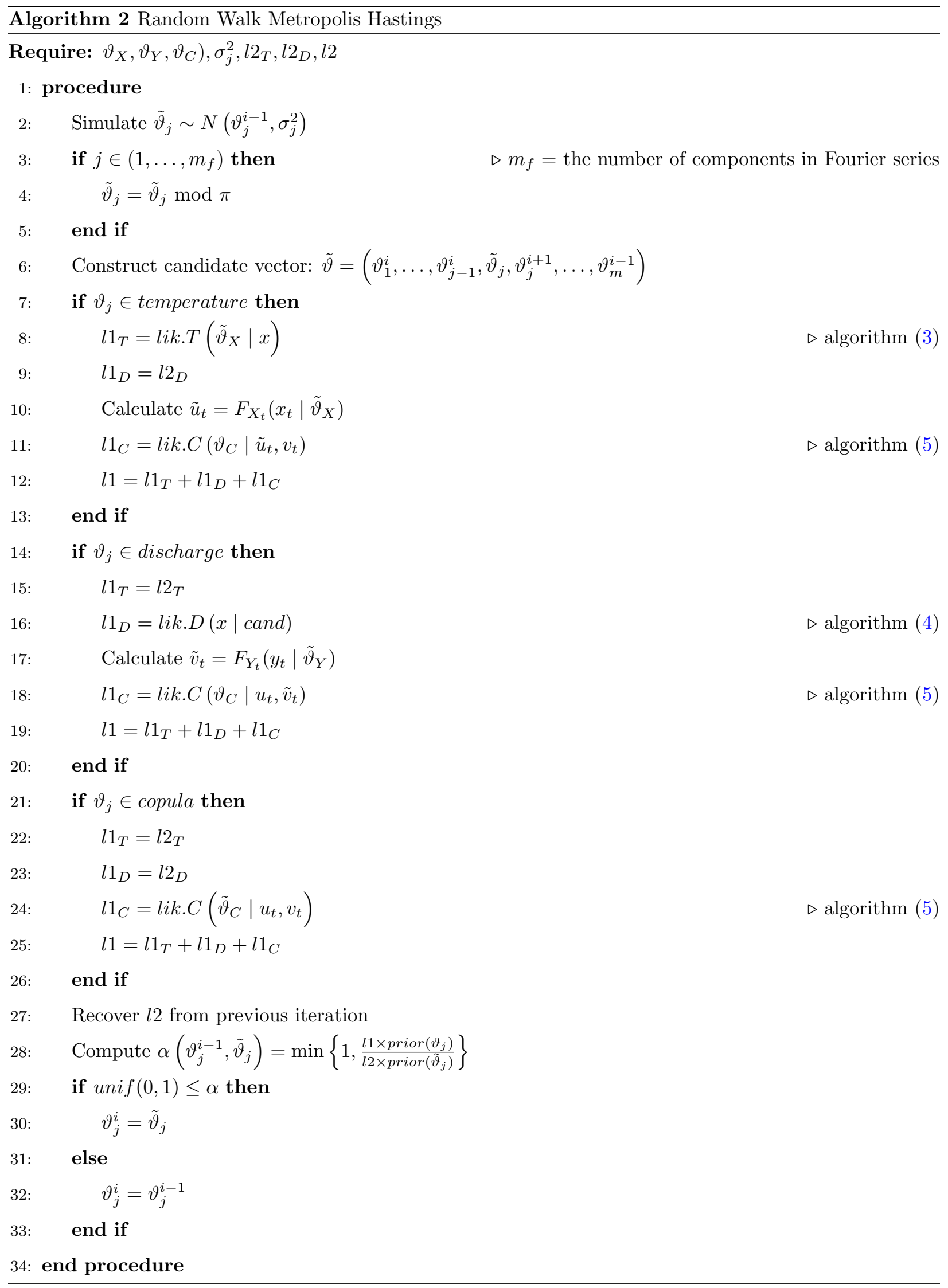



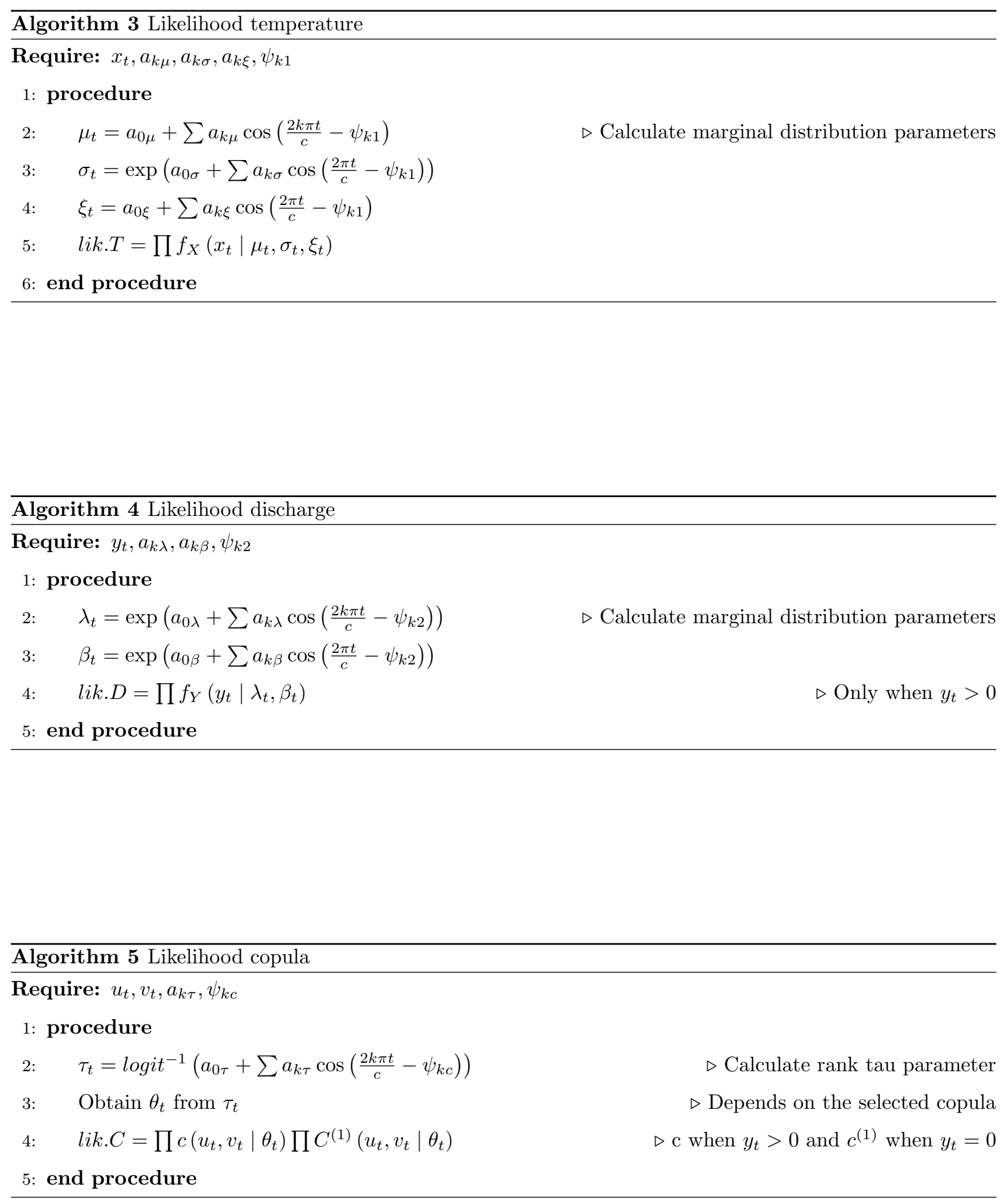


\section{References}

[1] Aas, K., Czado, C., Frigessi, A., Bakken, H., (2009). Pair-copula constructions of multiple dependence, Insurance: Mathematics and Economics 44 (2), 182-198.

[2] Ausín, M.C., Lopes, H.F., (2010). Time-Varying Joint distribution through copulas. Computational Statistic and Data Analysis, 54, 2383-2399.

[3] Azzalini, A., (1985). A class of distributions which includes the normal ones. Scand. J. Statist, 12, 171-178.

[4] Bers, A.V., Momo, F., Schloss, I. R., Abele, D. (2013). Analysis of trends and sudden changes in longterm environmental data from King George Island (Antarctica): relationships between global climatic oscillations and local system response. Climatic Change, 116, 789-803.

[5] Cantet, P., Arnaud, P. (2014). Extreme rainfall analysis by a stochastic model: impact of the copula choice on the sub-daily rainfall generation. Stochastic Environmental Research and Risk Assessment, Volume 28, Issue 6, pp 1479-1492

[6] Cogley, J.G., Hock, R., Rasmussen, L.A., Arendt, A.A., Bauder, A., Braithwaite, R.J., Jansson, P., Kaser,G., Mller, M., Nicholson, L., Zemp, M., (2011). Glossary of Glacier Mass Balance and Related Terms, IHP-VII Technical Documents in Hydrology No. 86, IACS Contribution No. 2, UNESCO-IHP, Paris.

[7] Cong, R., Brady, M. (2012). The interdependence between rainfall and temperature: Copula analyses. The Scientific World Journal, vol. 2012.

[8] Czado, C., (2010). Pair-copula constructions of multivariate copulas. In: Durante, F., Härdle, W., Jaworki, P., Rychlik, T. (Eds.), Workshop on Copula Theory and its Applications, Springer, Dortrech.

[9] Domínguez, M. C., Eraso, A., (2007). Substantial changes happened during the last years in the icecap of King George, Insular Antactica. In: Tyk, A., Stefaniak, K. (eds.): Karst and Cryokarst, Studies of the Faculty of Earth Sciences, University of Silesia 45, 87-110.

[10] Embrechts, P., Klüppelberg, C., Mikosch, T. (1997). Modelling extremal events for insurance and finance, Berlin: Springer Verlag.

[11] Embrechts, P., Lindskog, F., McNeil, A., (2001). Modelling Dependence with Copulas and Applications to Risk Management. Handbook of Heavy Tailed Distributions in Finance, ed. S. Rachev, Elsevier, Chapter 8, pp. 329-384.

[12] Genest, C., Favre, A. C., (2007). Everything you always wanted to know about copula but were afraid to ask, Journal of Hydrology Engineering, 4, 347-368. 
[13] Hock, R., (2003). Temperature index melt modelling in mountain areas. Journal of Hydrology, 282, 104-115.

[14] Hock, R. (2005). Glacier melt: A review of processes and their modelling. Progress in Physical Geography, 29, 362-391

[15] Hock, R., Jansson, P., Braun, L. (2005). Modelling the response of mountain glacier discharge to climate warming. In: Huber, U. M., Bugmann, H. K. M., Reasoner, M. A., Global Change and Mountain Regions - A State of Knowlegde Overview, Springer, Dordrecht, The Netherlands.

[16] Jansson, P., Hock, R., Schneider, T., (2003). The concept of glacier storage: a review. Journal of Hydrology, 282, 116-129.

[17] Kazianka, H., Pilz, J., (2010). Copula-based geostatistical modeling of continuous and discrete data including covariates. Stochastic Environmental Research and Risk Assessment, 24, 661-673.

[18] La Frenierre, J., Mark, B. G., (2014). A review of methods for estimating the contribution of glacial meltwater to total watershed discharge. Progress in Physical Geography, 38(2), 173-200.

[19] Nelsen, R. B. (2006), An introduction to Copulas, Second Edition. Springer, New York.

[20] Nazemi, A., Elshorbagy, A., (2012). Application of copula modelling to the performance assessment of reconstructed watersheds. Stochastic Environmental Research and Risk Assessment, Volume 26, Issue 2, 189-205

[21] Osmanoglu, B., Braun, M., Hock, R., Navarro, F. J. , (2013). Surface velocity and ice discharge of the ice cap on King George Island, Antarctica. Annals of Glaciology, 54(63),

[22] Ohmura, A., (2001). Physical basis for the temperature-based melt-index method. Journal of Applied Meteorology, 40, 75361 .

[23] Patton, A., (2012). A Review of Copula Models for Economic Time Series. Journal of Multivariate Analysis, 110, 4-18.

[24] Pellicciotti, F., Brock, B., Strasser, U., Burlando, P., Funk, M., Corripio, J., (2005). An enhanced temperature-index glacier melt model including the shortwave radiation balance: development and testing for Haut Glacier dArolla, Switzerland. Journal of Glaciology, 51, 573587.

[25] Rückamp, M., Braun, M., Suckro, S., Blindow, N., (2011). Observed glacial changes on the King George Island ice cap, Antarctica, in the last decade. Global and Planetary Change, Volume 79, Pages 99-109

[26] Saad, C., St-Hilaire, A., El Adlouni, S., Gachon, P., (2014). A nested multivariate copula approach to hydrometeorological simulations of spring floods: the case of the Richelieu River (Quebec, Canada) record flood. Stochastic Environmental Resources Risk Assess, 29:275-294. 
[27] Scholzel, C., Friederichs, P., (2008). Multivariate non-normally distributed random variables in climate research - introduction to the copula approach. Nonlinear Processes in Geophysics, 15, 761-772

[28] Spiegelhalter, D. J.; Best, N. G.; Carlin, B. P.; Van der Linde, A., (2002). Bayesian measures of model complexity and fit (with discussion). Journal of the Royal Statistical Society, Series B, 64 (4): 583-639.

[29] Tierney, L., (1994). Markov chains for exploring posterior distributions. Annals of Statistics, 22, 1701 1762.

[30] Toews, M.W., Whitfield, P. H., Allen, D. M., (2007), Seasonal statistics: The 'seas' package for R. Computers 83 Geosciences, 33, 944-951.

[31] Venter, G., (2001). Tails of copulas. In Proceedings ASTIN, Washington, USA, pages 68-113.

[32] Warburton, J., Fenn, C. R., (1994). Unusual flood events from an Alpine glacier: observations and deductions on generating mechanisms. Journal of Glaciology, vol.40, Issue 134, pp.176-186

[33] Willis, I.C., Arnold, N.S. and Brock, B.W. (2002). Effect of snowpack removal on energy balance, melt and runoff in a small supraglacial catchment. Hydrological Processes, 16, 272149.

[34] Zhang, Q., Xiao, M., Singh, V., Chen, X., (2013). Copula-based risk evaluation of hydrological droughts in the East River basin, China. Stochastic Environmental Research and Risk Assessment, Volume 27, Issue 6, pp 1397-1406. 